\title{
Numerical Study of the Characteristics \\ of Heat Pipes in the Composition Electronic Equipment of Spacecraft
}

\author{
Vladimir A. Kulagin*a and Nikita Y. Sokolov ${ }^{\mathrm{a}, \mathrm{b}}$ \\ ${ }^{a}$ Siberian Federal University \\ 79 Svobodny, Krasnoyarsk, 660041, Russia \\ ${ }^{b} \mathrm{AO}$ «Information Satellite Systemsthem \\ Academician M.F. Reshetnev» \\ 52 Lenin Str., Zheleznogorsk, 662972, Russia
}

Received 11.10.2013, received in revised form 23.03.2014, accepted 21.10.2014

T-shaped planar heat pipe (HP) is designed for use in the construction of spacecraft (SC). The article presents the developed mathematical model for calculating the thermal regime of the product using flat heat pipes, based on the solution of two-dimensional stationary equations of a of mass conservation, supplemented Darcy equations for fluid and vapor. For a given location heat sources and sinks on the surface of the flat heat pipe calculated distribution of pressure and mass flows for both phases of the coolant. Analysis of of working capacity of the heat pipe to the set conditions based on verification of the capillary restriction.

Keywords: flat heat pipe, spacecraft, mathematical model, heat transfer fluid.

DOI: 10.17516/1999-494X-2015-8-6-769-773.

(c) Siberian Federal University. All rights reserved

* Corresponding author E-mail address: v.a.kulagin@mail.ru 


\title{
Численное исследование характеристик тепловых труб \\ в составе радиоэлектронного оборудования \\ космических аппаратов
}

\author{
В.А. Кулагин ${ }^{\mathrm{a}}$, Н.Ю. Соколов ${ }^{\mathrm{a}, \boldsymbol{\sigma}}$ \\ ${ }^{a}$ Сибирский федеральнылй университет \\ Россия, 660041, Красноярск, Свободньй, 79 \\ ${ }^{6}$ АО «Информационные спутниковые системы \\ им. академика М.Ф. Решетнёва» \\ Россия, 662972, Железногорск, ул. Ленина, 52
}

T-образная плоская тепловая труба (ТT) предназначена для использования в конструкциях космических аппаратов (КА). В статье изложена разработанная математическая модель для расчета теплового режима работы изделий с использованием плоских тепловых труб, основанная на решении двумерных стационарных уравнений сохранения массы, дополненных уравнениями Дарси для жидкости и пара. Для заданного расположения источников и стоков тепла на поверхности плоской тепловой трубы рассчитаны распределения давлений и потоков массы для обеих фаз теплоносителя. Анализ работоспособности тепловой трубы для заданных условий основан на проверке выполнения капиллярного ограничения.

Ключевые слова: плоская тепловая труба, космические аппараты, математическая модель, теплоноситель.

Разница в давлении между жидкой и газообразной фазами теплоносителя в каждой точке трубы не должна превышать величины капиллярного напора, который способна развивать пористая структура [1].

Для жидкой фазы теплоносителя уравнение будет иметь вид

$$
\operatorname{div}\left(\beta_{l} \cdot \nabla P_{l}\right)=q_{e v} / H_{e v}
$$

где $\beta_{l}=d \cdot S_{l} \cdot K \cdot \frac{\rho_{l}}{\mu_{l}}-$ коэффициент в виде диагональной матрицы, отражающий гидравлическое сопротивление пористой структуры для жидкости; $d$ - толщина тепловой трубы; $K$ - проницаемость фитиля; $\rho_{l}$ и $\mu_{l}-$ плотность и вязкость теплоносителя соответственно; $P_{l}(x, y)$ - давление теплоносителя; $q_{e v}(x, y)$ - заданное распределение мощности тепловыделения по поверхности тепловой трубы; $H_{e v}$ - скрытая теплота парообразования теплоносителя; матрица $S_{l}=\left(\begin{array}{cc}S_{l x} & 0 \\ 0 & S_{l y}\end{array}\right)$ определяет долю эффективного сечения фитиля от общего сечения тепловой трубы для продольного направления $x$ (компонент $S_{l x}$ ) и поперечного $y$ (компонент $S_{l y}$ ) соответственно.

Решением уравнения (1) и аналогичного уравнения для пара являются распределения давления теплоносителя в паровой и жидкостной фазах по плоскости тепловой трубы. Разность давлений между фазами $\Delta P(x, y)=P_{v}(x, y)-P_{l}(x, y)$ не должна превышать величину капиллярного напора $P_{c}=2 \sigma \cdot \cos (\theta) / r_{c}$, где $r_{c}$ - радиус пор фитиля; $\sigma$ - коэффициент поверхностного натяжения; $\theta$ - предельный угол смачивания материала фитиля теплоносителем.

Дифференциальное уравнение (1) является параболическим, которое целесообразно решать численным методом. Методы решения таких уравнений широко известны, например, можно использовать метод простой итерации с Чебышевским набором параметров [0;0].

$$
-770-
$$


Т-образная плоская тепловая труба или гипертеплопроводящая секция (ГТПС) - это пакет мини-тепловых труб, заключенных в одном герметичном корпусе. Поэтому целесообразно сравнить математическую модель, разработанную для ГТПС, с математической моделью цилиндрических ТT.

Здесь предложена математическая модель теплопереноса в ТТ в квазитрехмерной постановке [2]. Задача сводится к решению системы уравнений (2)-(4), описывающих теплоперенос в тепловой трубе в цилиндрической системе координат по радиальной и угловой координатам, с осью симметрии, совпадающей с осью ТТ. Система уравнений включает двумерные нестационарные уравнения теплопроводности (2), (3) для жидкой и паровой фаз хладагента, а также двумерное нестационарное уравнение теплопроводности (4) для корпуса ТТ (рис. 1). Перенос тепла вдоль трубы по паровому каналу, фитилю и корпусу ТТ учтен в виде «источникового» члена в уравнениях (2)-(4):

$$
\begin{aligned}
& \frac{\partial T_{1}}{\partial t}=a_{1}\left(\frac{\partial^{2} T_{1}}{\partial r^{2}}+\frac{1}{r} \cdot \frac{\partial T_{1}}{\partial t}+\frac{1}{r^{2}} \cdot \frac{\partial^{2} T_{1}}{\partial \theta^{2}}\right)-\varphi_{1} \cdot \frac{U \Delta T_{m p}}{L_{m p}} \quad 0<r<r_{1}, 0<\theta<\pi \\
& \frac{\partial T_{2}}{\partial t}=a_{2}\left(\frac{\partial^{2} T_{2}}{\partial r^{2}}+\frac{1}{r} \cdot \frac{\partial T_{2}}{\partial t}+\frac{1}{r^{2}} \cdot \frac{\partial^{2} T_{2}}{\partial \theta^{2}}\right)-\varphi_{2} \cdot \frac{\lambda_{2} \Delta T_{m p}}{C_{2} \rho_{2} L_{m p}{ }^{2}} \quad r_{1}<r<r_{2}, 0<\theta<\pi \\
& \frac{\partial T_{2}}{\partial t}=a_{2}\left(\frac{\partial^{2} T_{2}}{\partial r^{2}}+\frac{1}{r} \cdot \frac{\partial T_{2}}{\partial t}+\frac{1}{r^{2}} \cdot \frac{\partial^{2} T_{2}}{\partial \theta^{2}}\right)-\varphi_{2} \cdot \frac{\lambda_{2} \Delta T_{m p}}{C_{2} \rho_{2} L_{m p}{ }^{2}} \quad r_{1}<r<r_{2}, 0<\theta<\pi,
\end{aligned}
$$

где $\varphi$ - доля площади поперечного сечения элемента ТT; $U$ - продольная составляющая скорости движения пара по координате z, м/с; $C$ - изобарная теплоемкость, Дж/(кг·К); $\theta$ - угловая координата, град; $\lambda$ - коэффициент теплопроводности, Вт/(м.К); $\rho$ - плотность, кг $/ \mathrm{M}^{3} ; t-$ время, с; $\Delta T_{m p}$ - перепад температуры по продольной координате, $K ; L_{m p}-$ длина тепловой трубы, м. Индексы: 1 - паровая фаза; 2 - зона фитиля; 3 - корпус тепловой трубы.

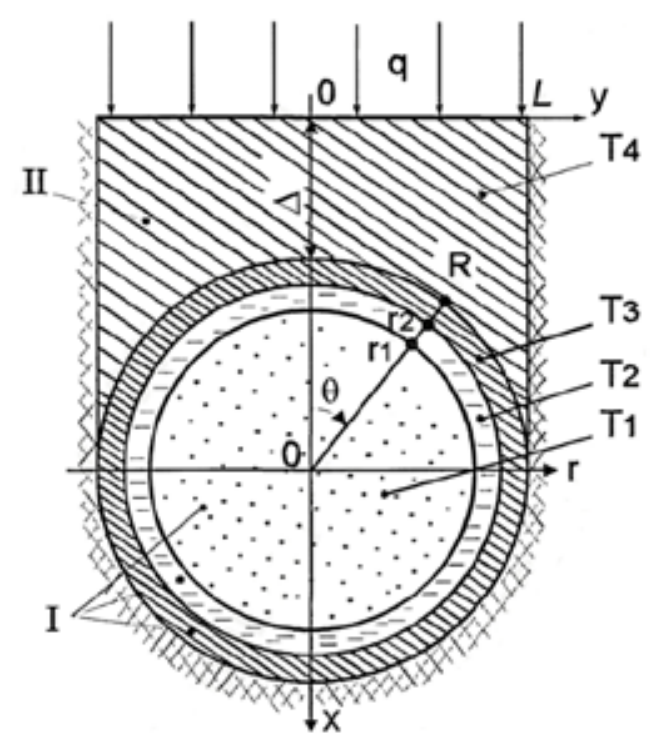

Рис. 1. Схема поперечного сечения системы «источник тепловыделения - соединительный элемент тепловая труба»: где I - тепловая труба, II - соединительный элемент, $q$ - подводимый тепловой поток 
Теплоперенос в соединительном элементе описан двумерным нестационарным уравнением теплопроводности (5) в декартовой системе координат:

$$
C_{4} \rho_{4} \frac{\partial T_{4}}{\partial t}=\lambda_{4}\left(\frac{\partial^{2} T_{4}}{\partial x^{2}}+\frac{\partial^{2} T_{4}}{\partial y^{2}}\right) \quad 0<x<\Delta+R, 0<y<L .
$$

Корпус трубы и соединительный элемент принимали изготовленными из одного материала с высокой теплопроводностью или из различных материалов с разными теплофизическими характеристиками [2].

При задании начальных условий при решении задачи в квазитрехмерной постановке температура в начальный момент времени распределена равномерно:

$$
t=0, T_{1}=T_{0}, T_{2}=T_{0}, T_{3}=T_{0}, T_{4}=T_{0} .
$$

В тепловой трубе описан теплоперенос за счет теплопроводности и учтен процесс испарения. Граничные условия в данной постановке:

$$
\begin{aligned}
& r=0 \quad \frac{\partial T_{1}}{\partial r}=0 \\
& r=r_{1}, 0 \leq \theta \leq \pi-\lambda_{1} \frac{\partial T_{1}}{\partial r}=-\lambda_{2} \frac{\partial T_{2}}{\partial r}-Q \cdot W, T_{1}=T_{2} \\
& r=r_{2}, 0 \leq \theta \leq \pi \quad-\lambda_{2} \frac{\partial T_{2}}{\partial r}=-\lambda_{3} \frac{\partial T_{3}}{\partial r}, T_{2}=T_{3} \\
& r=R, 0 \leq \theta \leq \frac{\pi}{2} \quad-\lambda_{3} \frac{\partial T_{3}}{\partial r}=-\lambda_{4} \frac{\partial T_{4}}{\partial n}, T_{3}=T_{4} \\
& r=R, \frac{\pi}{2} \leq \theta \leq \pi \quad \frac{\partial T_{3}}{\partial r}=0 \\
& x=0,0 \leq y \leq L \quad-\lambda_{4} \frac{\partial T_{4}}{\partial x}=q \\
& y=0,0 \leq x \leq \Delta \quad \frac{\partial T_{4}}{\partial y}=0 \\
& y=L, 0 \leq x \leq \Delta+R \quad \frac{\partial T_{4}}{\partial y}=0 \\
& \theta=0,0 \leq r \leq r_{1} \quad \frac{\partial T_{1}}{\partial \theta}=0 \\
& \theta=0, r_{1} \leq r \leq r_{2} \quad \frac{\partial T_{2}}{\partial \theta}=0 \\
& \theta=0, r_{2} \leq r \leq R \quad \frac{\partial T_{3}}{\partial \theta}=0 \\
& \theta=\pi, 0 \leq r \leq r_{1} \quad \frac{\partial T_{1}}{\partial \theta}=0 \\
& \theta=\pi, r_{1} \leq r \leq r_{2} \quad \frac{\partial T_{2}}{\partial \theta}=0 \\
& \theta=\pi, r_{2} \leq r \leq R \quad \frac{\partial T_{3}}{\partial \theta}=0 .
\end{aligned}
$$


Массовая скорость испарения хладагента рассчитывалась по формуле

$$
W=\frac{A\left(P^{H}-P\right)}{\sqrt{2 \pi R_{0} T_{\text {2.p. }} / M}},
$$

где $A$ - коэффициент аккомодации; $P$ - давление, Па; $R_{0}$ - универсальная газовая постоянная, Дж/(моль-К); $M$ - молекулярный вес, кг/моль. Индексы: г.ф. - граница фаз; н - насыщенный.

Давление насыщенных паров определили методом Риделя - Планка - Миллера [3]:

$$
\begin{aligned}
& \ln P_{v p}=-\frac{G}{T_{r}}\left[1-T_{r}^{2}+k\left(3+T_{r}\right)\left(1-T_{r}\right)^{3}\right] \\
& G=0,4835+0,4605 h \\
& k=-\frac{\frac{h}{G}-\left(1+T_{b_{r}}\right)}{\left(3+T_{b_{r}}\right)\left(1-T_{b_{r}}\right)^{2}} \\
& h=T_{b_{r}} \cdot \frac{\ln P_{c}}{1-T_{b_{r}}} ; \quad T_{b_{r}}=\frac{T_{b}}{T_{c}},
\end{aligned}
$$

где $T_{b}$ - нормальная температура кипения; $T_{c}$ - критическая температура, $\mathrm{K} ; P_{c}-$ критическое давление, атм.

Сформулированная система уравнений (2)-(5) с соответствующими начальными (6) и граничными условиями (7)-(20) решена методом конечных разностей [4]. Дифференциальные уравнения в частных производных (2)-(5) были представлены в виде разностных двумерных уравнений. Переход на новый временный слой реализован с помощью двух «дробных шагов» по схеме расщепления [4]. Систему одномерных разностных уравнений решали с помощью метода прогонки по неявной четырехточечной разностной схеме, обладающей абсолютной устойчивостью и хорошо себя зарекомендовавшей при решении задач теплопроводности [4].

\section{Список литературы}

[1] Деревянко В.А., Нестеров Д.А., Косенко В.Е. и др. // Вестник СибГАУ. 2013. № 6(52). C. 111-116.

[2] Колоусова А.А. Математическое моделирование теплопереноса в системе «источник тепловыделения - соединительный элемент - тепловая труба». Томск, 2004.

[3] Рид Р., Прауснии Дж., Шервуд Т. Свойства газов и жидкостей. Л.: Химия, 1982.

[4] Пасконов В.М., Полежаев В.И., Чудов Л.А. Численное моделирование процессов теплои массопереноса. М.: Наука, 1984. 288 с. 